\title{
Magnetic Induction Technology-Based Wireless Sensor Network for Underground Infrastructure, Monitoring Soil Conditions, and Environmental Observation Applications: Challenges and Future Aspects
}

\author{
Pratap Singh, ${ }^{1}$ Rishi Pal Singh, ${ }^{1}$ Yudhvir Singh, ${ }^{2}$ Jasgurpreet Singh Chohan ${ }^{(D},{ }^{3}$ \\ Shubham Sharma $\oplus^{1,3,4}$ Milad Sadeghzadeh $\mathbb{D}^{5},{ }^{5}$ and Alibek Issakhov ${ }^{6}$ \\ ${ }^{1}$ Department of Computer Science and Engineering, Guru Jambheshwar University of Science \& Technology, Hisar 125001, India \\ ${ }^{2}$ Department of Computer Science and Engineering, Maharshi Dayanand University, Rohtak 124001, India \\ ${ }^{3}$ Department of Mechanical Engineering, Chandigarh University, Gharuan 140413, India \\ ${ }^{4}$ Department of Mechanical Engineering, I.K. Gujral Punjab Technical University, Jalandhar-Kapurthala Highway, VPO Ibban, \\ 144603, India \\ ${ }^{5}$ Department of Renewable Energy and Environmental Engineering, University of Tehran, Tehran, Iran \\ ${ }^{6}$ Faculty of Mechanics and Mathematics, Department of Mathematical and Computer Modelling, Al-Farabi Kazakh \\ National University, Almaty, Kazakhstan
}

Correspondence should be addressed to Shubham Sharma; shubham543sharma@gmail.com and Milad Sadeghzadeh; milad.sadeghzadeh@gmail.com

Received 25 May 2021; Revised 16 August 2021; Accepted 9 December 2021; Published 7 January 2022

Academic Editor: Alfian Abdul Halin

Copyright (c) 2022 Pratap Singh et al. This is an open access article distributed under the Creative Commons Attribution License, which permits unrestricted use, distribution, and reproduction in any medium, provided the original work is properly cited.

\begin{abstract}
Wireless sensor networks (WSNs) especially with sensor nodes communicating with each other in medium other than air have been naive area of research since the last few years. In comparison to underwater communication, wireless underground sensor networks (WUSNs) are now being used in a large number of applications ranging from environmental observation, estimating chances of earthquake, communicating in underground tunnels or mines, and infrastructure monitoring to soil monitoring for agricultural purposes. In spite of all such promising applications, due to harsh and dynamically changing soil characteristics including soil type, water content in soil, and soil temperature, underground communication with conventional electromagnetic (EM) wave-based technology could not prove to be feasible for long-distance communication. Alternatively, due to magnetic permeability of soil being similar to air, magnetic induction- (MI-) based approach was adopted using magnetic coils as antenna for sensor nodes. Subsequently, MI waveguide and 3D coil mechanisms were considered to improve the system efficiency. Attributing to different characteristics of underlying transmission channels, communication protocols as well as architecture of MI-based WUSNS (MI-WUSNs) have been developed with different approaches. In this review paper, in addition to the latest advancements made for MI-WUSNs, closely associated areas of MI-WUSNs have also been explored. Additionally, research areas which are still open to be worked upon have been detailed out.
\end{abstract}

\section{Introduction}

The communication between sensing nodes in an underground medium is a difficult job, and researchers have been still trying to improvise it for the last more than four decades. This kind of communication helps in a number of application areas such as observing soil condition, predicting probability of earth quakes, and many more [1]. A number of sensors have been deployed under the ground surface for these applications. Therefore, the sensors are placed below the bottom surface. These sensors also constitute the screening architecture and may provide additional sensing 
information with more precision as compared to if those were deployed on and above the surface [2].

It is known that with the increase in count of sensors, measurement precision is also increased, which leads to using a greater number of sensor nodes. In a parallel manner, separate operational cost of every node ends up in large exploitation cost, which is generally not possible to be supported economically. Contrarily, it has been established by Akyildiz et al. [3] that coordinated effort of multiple sensor nodes organized in a network reduces the energy requirements and enhances the reliability of measurements. This will be attained using collectively processed information and transmission, respectively. Resultantly, the complexness of all nodes and their design cost is bound to decrease.

The last few years have witnessed underground wireless sensor networks (WSNs) having been used for numerous events and applications like those for the environment, health, military, home, and industry [4]. Akyildiz and Vuran [4] clearly describe the designing guidelines for all underlying communication layers. This paper also analyzes the usage of WSNs in tough and challenging environments. The study on how signals propagate through the soil for wireless underground sensor nodes (WUSNs) is investigated by [5]. They proposed a two-phased coefficient model, one for a near-field region and other for a far-field region. Akyildiz and Stuntebeck explained the usage of WUSNs for the underground applications [1]. Particularly, for the underground applications, wireless underground networks are projected in [1]. Authors have also discussed the benefits of using WUSNs such as easy deployment, no more collisions of sensors with landscaping objects and instruments like tractors, retrieval of information in real-time environment, self-healing capability in sensor failure case, and scalability of nodes $[1,3]$.

The underground transmission link, which is largely influenced by the heterogeneously varying soil medium, is one major factor coming in the way of the complete exploration and evolution of WUSNs. This underground medium consists of sand, rocks, and even watersheds. It has also been found that the soil humidity highly affects the transmission distance and therefore the net performance of the WUSNs. In numerous real-life examples, electromagnetic (EM) wave-based traditional wireless signal communication mode may only be used for very limited transmission distance, owing to significant path loss and dynamically changing soil conditions [6].

As a strong alternative of conventional EM communication, magnetic induction- (MI-) based communication mode has been taken into consideration. Work on MI-based communication has already been done under the purview of near-field communication (NFC) [7] and wireless power transfer (WPT) [8]. Contrary to EM wave-based communication, in MI-based communication, induction coils are used (also known as magnetic antennas) to produce a quasi-static magnetic field. The receiving coil will then detect such magnetic fields produced by the transmitting coil, and the corresponding signal transmission channel is set up.

MI-based WUSNs are initially described in [9] which showed that this technique leads to lower path losses in the conductive medium (soil), which will greatly increase the transmission range as well as the transceiver coverage area.

The prominent contributions of this paper are as follows:

(i) Having clarity about various categories of application domains where MI-WUSNs are a quite promising option to be used

(ii) Study of developments made in various areas related to MI-WUSNs so as to analyze their impact on implementation of MI-WUSNs

(iii) Analysis of recent advancements made in MIWUSNs to explore open challenges and future scope in this domain

This review paper has been structured as follows. Section 2 describes different categories of prominent application domains making WUSNs a promising solution. Section 3 details out the developments carried out in closely associated fields of MI-WUSNs ranging from EM-based WUSNs to magnetic field communication techniques and under water wireless communication. Section 4 highlights the various dimensions of communications in MI-WUSNs and the advancements in those respective areas. Section 5 gives SWOT analysis of EM- and MI-based WUSNs along with major protocols used for these technologies. Section 6 consists of inference and some new research challenges supposed to be addressed in the future. Section 7 presents the conclusion of the review.

\section{Application Domains of WUSNs}

The EM wave- and MI-based WUSNs differ from each with respect to physical layer mode and overall system performance affected by the physical communication channel. For EM-based WUSNs, due to different types of soils and other materials like rocks buried under the earth surface, signal path loss of EM waves is highly unpredictable due to different permeability of soil, water, and rocks. On the other hand, for MI-based WUSNs, magnetic permeability of soil or water or rock is the same as that of air, and therefore, these factors do not affect the signal path loss. For both EM- and MI-based WUSNs, the following are the prominent application domains of usage.

2.1. Observing Underground Infrastructures. WUSNs are majorly used to monitor the underground infrastructure including buildings, pipes, electrical wirings, and liquid storage tanks.

One important application of WUSN is to observe the health of infrastructures like residential or commercial buildings, if the parts of these infrastructures, like foundation, are connected with the soil. One may also monitor the support of infrastructures like bridges or dams with WUSNs [10]. Therefore, possible calamities may be prevented by observation of different concerned characteristics like stress or strain. It could prove to be more beneficial for structures deployed in nonstable media including water 
and sand, as the chances of nonstable slopes are quiet high in such locations [11].

Additionally, WUSNs may also prove to be beneficial to monitor underground plumbing, where sensor nodes may be put along the path of pipes in order to instantly detect, localize, and repair the leakage [12]. Underground fuel station tanks may also be monitored using the same mechanism, which continually determines the amount of fuel in tank. Also, for military applications related to underground infrastructure, such as minefield monitoring, WUSNs may be effective [1].

Moreover, WUSNs may monitor the structural conditions of undersurface coal mines, which will ensure protection of coal mine staff. Therefore, sensor nodes are required to be placed within the walls as well as ceiling of the gallery areas of mines to identify any structural disturbances. On a similar pattern, WUSN sensors may be placed along the side of the tunnels to monitor their structural health [2]. The environment-related parameters such as relative humidity, temperature, and gas concentration are important factors that are considered and sent to the base station, which is connected to the ground observation system. A WUSN-based fire monitoring system can take decision by observing this data to minimize the chances of fire in coal mines [13].

2.2. Monitoring Soil Conditions. The most prominent area of applying WUSNs is agriculture where sensor nodes may be buried in soil to observe the soil conditions such as composition of minerals and humidity, without causing any obstruction for objects like lawnmowers and tractors [1]. This approach leads to providing data for appropriate fertilization as well as irrigation. Underground WSNs are also used in a greenhouse setting where sensing nodes are deployed in the pot of each plant, thereby offering concealment.

Sports field monitoring [1] is also one promising application area of WUSNs, where sensor networks may be utilized to do monitoring of soil conditions at fields like golf courses, grass tennis courts, soccer fields, or baseball fields. For such type of sports, poor conditions of turf generally cause annoying playing experience, making it very essential to maintain the soil for ensuring good grass health.

One more application of WUSNs is to detect the presence as well as concentration of harmful substances, which may contaminate the soil near rivers and aquifers. For this purpose, the concept of a hybrid network has been recommended by Akyildiz and Stuntebeck, which might be deployed partly under the ground and partly under the water medium [1]. Wu et al. [14] proposed a long-range wide area network for agriculture WUSNs with very less path loss. The network connectivity covers a range over several kilometers because in agriculture, no frequent acknowledgement is required. Sambo et al. [15] applied a WUSN path loss model (PLM) for precision agriculture. WUSN-PLM works on accurate prediction of the complex dielectric constant. By considering the reflective and refractive wave attenuation, it evaluates the intensive measures of sensors.

2.3. Environmental Observation. It is also a possible application domain to monitor the air quality in underground coal mines for WUSNs [1]. It involves the composite architecture of open-air underground sensing nodes and embedded underground sensing nodes deployed between the ground surface and the mine tunnel roof. This allows data from mine sensors to be routed vertically to surface stations quickly, instead of getting routed through the large distances of tunnels in mine.

Earthquake and landslide prediction is also an important application. For this, the deeply buried sensors monitor the relative movement of the layers of soil, so as to predict the disasters with more accuracy and far earlier as compared to existing mechanisms [16]. Therefore, the population residing the affected areas may be timely warned and displaced. Also, the prediction may be more accurate if sensors are deployed at different depths in soil.

The applications related to glacier movements and volcanic eruptions being monitored by buried sensor nodes are elaborated in $[17,18]$, respectively. This option of deploying sensor nodes in WUSN architecture gives more precise observations and at the same time saves the sensors from damage caused by heat of volcano or movement of ice blocks of the glacier. Also, the smart insect pest detection has been proposed using WUSNs for precision agriculture. Figure 1 shows the interconnection of various sensors, fans, and monitoring units for monitoring and control of air pollution [19]. In this system, the direct digital control technology automatically switches on the ventilator fans once air quality deteriorates as signaled by the sensor.

2.4. Determining Location of Objects. Object localization is a prominent application as well as research area for WSNs where stationary underground sensing nodes aware of selflocation may be used as beacon for locating the required objects. Location information can serve as a navigational tool for self-driven systems; e.g., autonomous fertilizer units move on the field to be fertilized depending on underground location beacons and soil condition data from sensing nodes buried in soil.

Also, for underground sensor networks, locating animals or human beings comes out to be one of the major applications [2], specifically when unexpected calamities happen in fields described in above sections such as earthquake, land sliding, and cave-ins. Assuming the deployment of WUSNs to be in several risky areas with requirement of sensors still being functional even after disaster, the cooperative effort of sensing nodes may be useful in localizing staff persons trapped in mines or people in a crumpled building. For this, each individual within the mine or building should be wearing appropriate devices, which might transfer a beacon signal and thereby interact with the WUSNs. Therefore, the position of the person trapped may be determined and informed to the search team, which makes the search operations far easier and hence protects human beings.

Another important application area of the localization by WUSNs is to locate small creatures like badgers or rabbits in their natural habitat, in order to understand their behavior as well as evolution process [2].

Additionally, for the purpose of navigating the autonomous vehicle, the beacon signals from underground sensors 


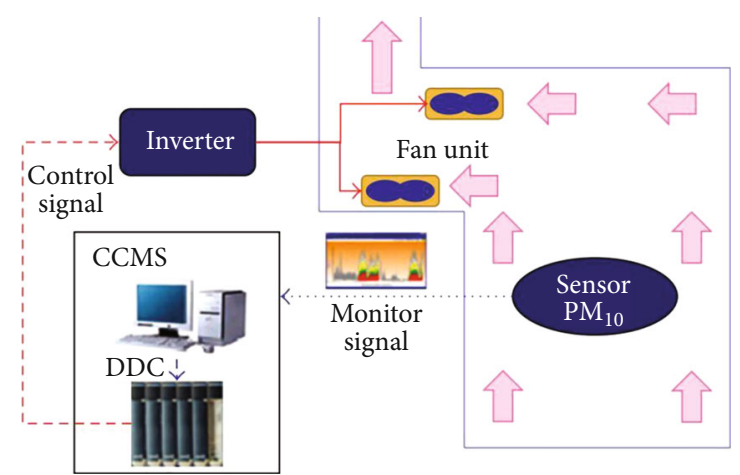

Figure 1: Ventilator fans and control systems used to monitor air quality [19].

put within the road concrete may be used. In coming days of more and more self-driving vehicles, it is going to be more promising application of WUSNs.

In law enforcement and security, optical sensors play a critical role. Night vision scopes for border patrol and surveillance, motion sensors for home security alarms, and physical specimen detection at crime scenes all employ them. Border patrolling and security observation may be used to detect the presence and movement of persons or objects above the surface. For such security-related applications, WUSNs may be placed to find invaders or interlopers and warn the security organization. Akyildiz and Stuntebeck described that it is quite difficult to detect the presence of sensing nodes buried inside the soil [1]. Moreover, the invaders are less likely to discover and disable the security system. Correspondingly, WUSNs may be put along the border side to identify the unauthorized crossings and to inform the concerned authorities. Here, crossing detection with high precision by WUSNs could facilitate the authorities to simply localize the invaders.

\section{Allied Domains of MI-WUSNs and Their Recent Developments}

The study of prominent fields directly or indirectly allied to MI-based WUSNs facilitates the fruitful development of MIbased WUSNs. Such allied domains include EM wave-based underground WSNs, magnetic transmission-related technologies such as NFC or WPT, and underwater MI-based WSNs. The current advancements in these fields are partly applicable in implementation of MI-based WUSNs as well. Therefore, in this section, such developments are covered one by one to have a good idea about the relevant approach and system aspects, which could be incorporated in MIbased WUSNs in time to come. Figure 2 shows that the model is envisaged using Internet of underground things for providing total autonomy to agriculture activities [20]. This enables self-reporting of soil moisture, salinity, and temperature and hence ensures in situ monitoring of soil.

3.1. EM-Based Underground WSNs. Communication between sensing nodes buried in the underground medium has conventionally been EM-based and has been treated as a difficult task. Because of the conduction properties of the soil medium, the EM transmission faces the problem of very high attenuation [6]. Additionally, it is not a trivial job to develop a sensible but economical communication system. The core characteristics as well as challenges of designing EM-WUSNs are self-addressed in [1]. Particularly, the essential limitations of the underground sensor networks including dependability, concealment, and broadcasting are described. Also, the feasible solutions for enhancing the transmission capability, improved network, etc. are given in [1]. The multiple dimensions of system development for such networks have been analyzed in [6]. Despite the first conceptualization of the signal propagation in underwater as well as underground medium proposed by different researchers within the past, the channel models are deeply explained and extensively characterized in [21-23]. Additionally, empirical studies for underground-to-aboveground and underground-to-underground [24, 25] transmission capabilities are simplified. In the same context, the issue of multipath propagation in underground media is also addressed. Silva and Vuran [26] and Yu et al. [27] have supported some of the aspects of the present channel models through experimental data as well.

Salam and Vuran have very elaborately explained crosslayered network optimization for the WUSNs [28]. Moreover, Liu et al. [29] and Yuan et al. [30] have resolved the issues with constrained battery resources of WUSNs. In [29], the network development is taken into account for the data communication as well as for the recharging of the batteries of sensor nodes. Supplementary relay nodes are placed in definite positions, for maintaining the network connectivity and correspondingly for increasing the lifetime [30]. Because of reflection and scattering of signals in underground medium, the communication channel appears to produce a large degree of diversity that may be exploited by sensible antenna techniques [31, 32]. Similarly, by exploiting relay nodes, the lifetime of the WUSNs can also be improved. Tam et al. used two different algorithms for maximizing the network longevity [33]. A detailed analysis of underground sensors for WUSNs is made by Trang et al. [34]. This analysis compares the EM wave system and MI system qualitatively as well as quantitatively. As described in earlier sections, prominent application domains of WUSNs are of agricultural purpose and communication in tunnels [21] or mines [35]. Subsequent domain applications need multifaceted channel modelling because tunnel or mine infrastructure is substantially different from agriculture. Moreover, the invited case of information gathering supported by the central pivot system is thought in $[26,36]$.

3.2. Prominent MI Signal Transmission Techniques. In the last few years, magnetic induction- (known as magnetic resonance also, in case of resonance circuits) based transmission techniques have been more popular and also got place in the key communication standards followed in the modern electronic gadgets such as smartphones and tablets.

Most of the typical MI signal transmission-based applications do come under the category of the WPT mechanism and NFC mechanism. It is renowned that the NFC technique varies from conventional far-field communication 


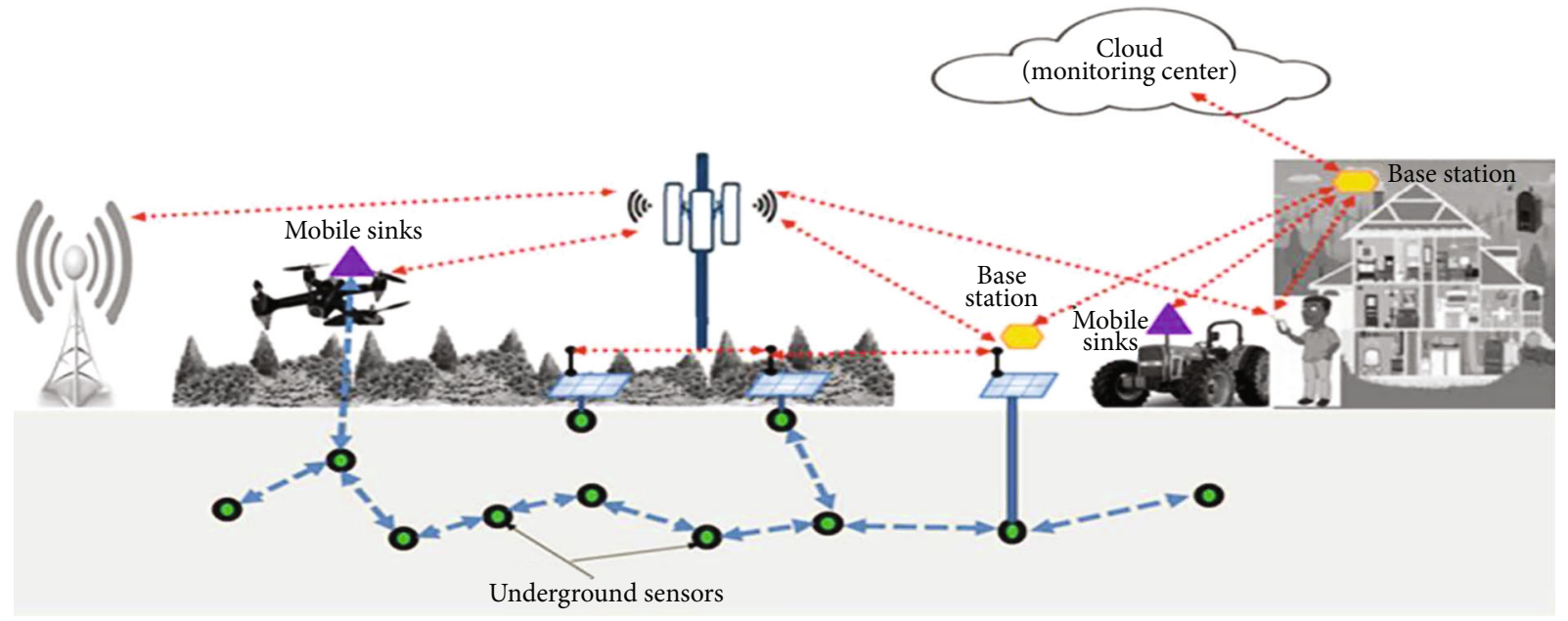

FIGURE 2: WUSN model for precision agriculture [20].

because of the nonlinear characteristics of the magnetic field, when several magnetic devices are located near to each other [2]. Therefore, the attempt to simplify the characteristics of the magnetic transmission channel did not provide adequate precision for system modelling. Also, a number of factors have been found to be depending upon count of magnetic nodes, their relative distances, and related system parameters. Therefore, with the increase in the number of nodes, the system development complexness is bound to increase, making it typical for many currently available communication mechanisms.

NFC is designed as a specialized kind of radio frequency identification (RFID) [37] for very small transmission distance less than $4 \mathrm{~cm}[7,38]$. Generic application areas for NFC include social networking, keycards, and contactless payment system [2]. In this, short-distance communication is seen as a major benefit of the MI transceivers, as it lessens the likelihood of eavesdropping [39, 40]. Finkenzeller [37] and Jing and Wang [41] investigated the channel model and its near-field effects in their research. Meanwhile, an NFC forum is also established to confer the latest advancements and standardization of the NFC.

The obtainable data rate of an NFC point-to-point communication is maximum $424 \mathrm{Kbps}$ that is based mostly on the passive RFID. The option of passive circuit components and few possible assumptions usually settle this data transfer rate [37]. For overwhelming this limitation, a straightforward NFC link is built up by a multiple-input multipleoutput (MIMO) technique [42-44] and other cooperative relay devices $[45,46]$. Additionally, NFC may be a good option for the forthcoming IoT applications due to the bounded interference caused to the transceivers placed adjacent to each other $[47,48]$.

WPT within the near field is an exploration space for decades because of its high potency [49]. Some elementary work is provided in [50-52]. During the context, the impacts of the various transmission channels, e.g., frequency splitting [53], coil placement [54], and resistance matching [55], are investigated. In recent years, the concept of wirelessly charging the electrical vehicles and mobile phones has come up as compared to wire-based connection that reduces the movement of the wireless devices $[56,57]$.

Unfortunately, one can achieve high efficiency of the WPT for only limited distance of few centimeters, so that the coupling among the magnetic resonators may be significantly robust. Therefore, to enhance the communication range, the current point-to-point WPT has been extended by using multiple transmitter [58], multiple receiver [59], and few supplementary relay $[60,61]$ devices. Moreover, multiple transmitter and receiver nodes are arranged into generic MI-based MIMO schemes, which are investigated by [62-65]. In these works, many multiuser cases are taken and also the beam-forming coefficients of a colocated [63] or distributed [65] transmitter are optimized w.r.t. totally different parameters such as total receive power or minimum receive power among different receiver locations. Sun et al. [66] have analyzed the magnetic MIMO related to multiple transmitting and receiving nodes, where by using magnetic beaming, a secured WPT can be attained for the expected user. Meanwhile, many industrial solutions are offered, among them WiTricity has been considered one of the most popular solutions [67]. Zhao et al. [68] proposed a reinforcement learning-enabled efficient data gathering model for WUSNs by applying an optimal transmission policy by considering the factors like path loss of sensory data transmission, energy limitations, and network load balancing. In addition, the combination of WPT and NFC leading to the SWIPT principle sounds even more advantageous to the IoT [69]. In this mechanism, the same signal is employed for data as well as power transfer that is often more energyefficient as compared to ordered transmission.

3.3. Underwater MI-Based WSNs. Underwater communication could be a well-researched topic with different popular application areas like marine science and marine forces. Such application domains use signal propagation through the underwater medium, usually ocean water, which because of active salt ions as well as free electrons offers high electrical conductivity up to $4 \mathrm{~S} / \mathrm{m}$ [70]. Correspondingly, EM waves will go up to short distances and even at extremely 
low Hertz order signal frequencies. The effective bandwidth of the channel is normally even smaller. Generally, the underwater communication gets accomplished through acoustic waves that offer higher communication distance of several kilometers. But a large, tough, dynamically changing as well as frequency-selective communication channel is one major disadvantage of this system.

Preferably, MI-based signal communication is given in [48, 71]. Indeed, the MI transmission range is smaller as compared to acoustic waves. However, this disadvantage is covered up with stability of the system and transmission ease. In the recent works [72] on MI underwater sensor communication, the benefits of 3D coil antennas have been recommended to enhance the abstraction diversity and directivity of signal transmission. One more advice is to use multiple frequency bands [73], such that an extra degree of freedom is often exploited for the resource allocation that will increase the general attainable data rate. Moreover, an efficient magneto-inductive-based implementation has been described in [74].

Additionally, one more special area of application for MI-based communication is wireless communication in old reservoir tanks and pipelines, where leakage is detected by applying MI-WSNs $[12,75]$. For this purpose, Guo and Sun [76] worked, defined, and developed the channel and energy consumption models.

Unfortunately, such applications impose harder restrictions on the dimensions of the sensors, e.g., placing relatively smaller coils, than those of underground or underwater application areas. Additionally, the transmission range of every sensing node is significantly small and also the system layout is even higher challenging as compared to the layout of MI- (primarily) based underwater communication systems. The energy-efficient cross-layer resolution has been given in this respect in recent years [77].

Generally, MI-based communication in liquids is still an open problem because of high path loss and sophisticated transmission. The corresponding transmission channels, however, are almost identical to the MI-WUSN transmission channels, so that advances in one field can simply be incorporated in the other. Therefore, the new technique designed for MI-WUSNs is possibly utilized for the design of the tougher communication in the liquid medium. The next section will highlight various techniques for MI-based underground WSNs. Sambo et al. proposed a new path loss model by using a better model of CDC prediction as compared to existing path losses [15]. A good comparison of path loss models of radio frequency in soil is covered in [78]. In order to estimate the signal attenuation, two major models, i.e., modified Friis and CRIM-Fresnel, have been compared. In samples with lower permittivity values, the CRIM-Fresnel model gives a better attenuation estimate, whereas the modified-Friis model has greater accuracy in samples with higher clay or water content with higher permittivity values.

\section{Recent Advancements in MI-WUSNs}

Akyildiz et al. defined the need of MI transceivers for WUSNs with the regular advancement of research on MIbased communication systems [79]. The main concern here is that, contrary to the traditional approach focused on EM waves, MI communication is considered less infected during the modification in the state of the soil. The different paradigms used for MI-based communication have been demonstrated, where multiple nodes interact together with MI as underlying physical medium. The sink node is connected to a gateway, where the data is first accumulated and then uploaded to a server for further processing. The prime focus of this study is on MI-WUSNs, which is the component of the network which is deployed within the underground medium, as classical techniques are used to establish surface communication. The following sections detail out advancements on various aspects of MI-based WUSNs for better understanding.

4.1. Channel Modelling. An induction coil is the main element of each MI transceiver. It creates quasi-static magnetic field around itself that is detected by another MI transceiver's coil. In addition, to decrease the path loss at chosen resonance frequency and to create the circuit resonant, a capacitor is attached to each coil. Due to the comparatively higher transmission range $(10-100 \mathrm{~m})$ between sensors of MI-WUSNs, coils having adequate size are generally used, e.g., solenoids with a radius of $12-25 \mathrm{~cm}$ [80]. It is different from NFC in that small coils of radius of a few centimeters have been used, providing a much narrower transmission range.

A larger path loss exponent (generally 6 in free space) in comparison with classical EM waves (generally varies from 2 and 4 according to the condition) may be an essential characteristic for this form of communication [2]. Because of the soil as propagation medium being electrically connective, the magnetic field in soil has been weakened due to the simple eddy current affairs [81]. The primary flux from Tx. coil therefore yields slight currents, which in turn generates secondary magnetic fields which repel primary field and reduces the MI. With increasing signal frequency, this effect is reinforced. For heterogeneous medium, distinct layers of propagation medium with dissimilar conductivity level are expected.

Considering the location of the coil with respect to the substances of medium, magnetic fields are interpreted by the number of methods. For a transmitter coil established in soil (between 2 mediums showing completely dissimilar conductivities), the multiplying factor is computed in [82]. If the coil has been placed deeply in earth, then signal propagates via the underlying heterogeneous medium with numerous layers as analyzed in [83], where identical skin depth of the different media is concluded that correlates the average of the skin depths among all the layers.

Due to increased path loss, low resonance frequency between $100 \mathrm{~Hz}$ and $1 \mathrm{MHz}$ has been preferred for MIWUSNs with transmission distances more than 10 meters. Kisseleff et al. [84] showed the optimum resonance frequency decreased by the square of distance among the transceivers. However, despite of the optimum resonance frequency, the path loss proportionate always greater than the 10th power of the space, which distributes signal transmissions over huge ranges (more than one hundred meters), is not possible.

The basic classification of the magnetic flux propagation within the underground medium is covered by Wait [82]. 
The model of the MI transceivers along with their channel modelling is discussed in [9]. Latterly, all characteristics and features of system and network modelling are restudied, as MI-based transmission media vary considerably from conventional EM wave-based communication media. Also, the path loss of a point-to-point communication link is broadly represented in $[9,85]$.

A recent variety of asymmetric transceivers for largerange communications in soil is described in [86] to control the property even in the case of expected arrangement. Here, multiple tiny receiving coils, and distinct huge transmitting coils are connected to every sensor node. Using this, the signals are often acknowledged by at least one coil of the transceiver even during the arrangement; thus, the chance of interruption has been reduced. But, the relevance of such multicoil transceivers still needs a careful analysis.

The effectiveness of single MI link and the complete MI WUSNs is widely studied by applying the derived channel models for point-to-point signal communication.

4.2. Single MI Link. The researchers have analyzed the impact of various system parameters on point-to-point communication channels [80, 87-89]. In particular, the effects of various types of minerals at the signal propagation and system efficiency have been explored in [87]. On the other hand, authors concentrated on the improvement of coil arithmetic and resonance frequency $[89,90]$. Moreover, the system performance in terms of data transfer rate is demonstrated in [9] that is acceptable for the target operations of the WUSNs, motivating us for all new research. It is noticed that the data transfer rate of up to $1 \mathrm{Mbps}$ is offered by MI, even for fair transmission ranges at most fifteen meters and eight meters in dry and wet soil, respectively. Additionally, conventional EM radiation does not offer acceptable data rates for transmission ranges more than five meter even in dry soil. Additionally, sensible data communication techniques are explored for MI-WUSNs and demonstrate the control losses compared to the performance bound [83].

Additionally, developments in the field of magnetic metamaterials [91] have facilitated the utilization of presumed MI waveguides (relaying of multiple passive MI devices and metamaterial-improved MI transceivers) [92]. These advanced MI transceiver configurations can be used to achieve the better directivity of signal propagation that is highly low with the conventional induction coils. In particular, the characteristics of every passive relay among MI waveguides are improved w.r.t. the bandwidth efficiency [80] and generally attainable data rates. The limited manufacturing and inefficient deployments are the major drawback of MI-WUSNs, which can render its high cost.

Preferably, the usage of conventional (active) relaying schemes looks highly promising [84], as it has depended on the arrangement of the restricted number of extra relay controllers, whereas general data transfer rate is improved by up to an order of magnitude compared to the basic scenario with no relays.

Generally, comparatively high resonance frequencies varying within $1 \mathrm{MHz}$ and $20 \mathrm{MHz}$ have been found desirable for relay-based MI-WUSNs because of the limited transmis- sion range of every sublink. Moreover, the frequency selection of the channel strongly relies upon the quantity of MI transceivers and relays, as every device behaves like a narrow band pass filter irrespective of its major performance. It is often caused by using resonance circuits [80].

The combined usage of active and passive relays is an encouraging extension of the already available MI-WUSN solutions. In particular, in terms of WPT, MI waveguides constructed by strong couplings among neighboring relays often appear extremely useful where high bandwidths are not essential. MI waveguides can therefore be useful for charging the network [2].

4.3. Synchronization and Channel Estimation. Synchronization is not often covered within the theoretical works on MI-WUSNs, as the popular synchronization techniques when put to MI-WUSNs have not shown impact considerably as compared to the efficiency of synchronization of conventional complicated communication networks and sensor networks [93]. Furthermore, the synchronization of packet transmissions for communication networks is covered in [94] which corresponds to transmission scheduling in MI-WUSNs.

Alternatively, the evaluation of the magnetic transmission channel within the underground scheme is a useful exercise which is hardly addressed in few works [95]. Another useful finding is that frequency property of the transmission channel typically relies upon the resonance circuit and rarely on the range or the properties of semiconductive soil. Furthermore, here coupling changes the current in each circuit along with the transmitters [95]. The advantage of this method is that any specific training sequence is not required for the channel estimation, because the transmitted symbols are fully acknowledged. This method is adopted in [96], where a channel estimation protocol is proposed for bigger MI-WUSNs. This kind of channel assessment might not only permit automatic transmit power management but additionally permits the localization of nonworking devices, which may trigger the reconfiguration procedure and set up an alternate signal routing.

Apart from this work, it is often considered that a system designer and every MI transceiver are aware about the channel state information (CSI), assuming that deployment of WUSNs is stationary. Thus, the transmission channel almost always stands unaltered. Therefore, a channel assessment process with adequately long averaging is performed before the principal working of the WUSNs begins. Similarly, near to ideal CSI is accessible during the main activity. Still, upcoming approaches of system layout for MI-WUSNs should include the variation in the environment-related parameters that can change during unexpected earthquakes or precipitation [6]. Therefore, the system parameters may be chosen by the way of robust optimization as probability density of every taken environmental parameter.

4.4. Strategies of Deploying Sensor Nodes. The distribution of sensing nodes among the selected region of significance is a crucial condition of the network layout. The various ways of distribution of sensing nodes are proposed within the past, 
which use different measures and parameters. Particularly for MI-WUSNs, orientation of every coil as well as the position of the sensing modes is essential. Specifically, when magnetic field is not taken by a coil placed orthogonally to its polarization, direction of the coils may be changed to minimize cochannel interference between the network components [90]. In the MI waveguide, including several passive relays, the direction of coils may be chosen in such a way that the power leakages may be reduced for a defined frequency [97]. Therefore, the efficient bandwidth of the communication channel may be strengthened by improving the direction of every relay and the resonance frequency of every circuit. Furthermore, distribution method is associated with a considerably improved deployment work, because every coil requires to be put and directed with precision.

With an aim of minimizing the complexity of the ensuing system, authors have highlighted the two simplified distribution methods: "horizontal" and "vertical" distribution methods [88]. In "horizontal" distribution methods, the axes of every coil concentrate on the same direction of signal propagation, therefore enhancing the collective induction among the devices of the same connection. In the "vertical" distribution method, the axis of every coil highlights towards the bottom area, because quasi-omnidirectional relation propagation is attained. This method is highly beneficial for MI-WUSNs having passive relays. Thus, every device is linked with other node of the network $[88,90]$.

In MI waveguide-based WUSNs, the path highlighted by waveguides is the actual path of signal transmission. Thus, connection is not built between the nodes, until they are not linked by the way of MI waveguides. The logic exists within the selection of the signal frequency, which is often greater for networks built on MI waveguides. That frequency produces a high path loss for direct MI transmission; thus, only transmission through MI waveguides is possible. Similarly, the routing revolves around the selection of devices to be linked through MI waveguides. Therefore, these devices should be attentively chosen, so as to improve the performing network throughput or property [94, 98]. In [98], a Voronoi tessellation is used to choose the nodes to be linked via MI waveguides. A heuristic approach is applied, which relies on recursive calculation of an MST and omission of suspected links. In addition, the degree of distribution is an effective characteristic of the system architecture. It is certain that if two coils are placed above the ground surface close to each other, a portion of the produced field will propagate through the air, while another portion propagates through the soil [88]. Therefore, in comparison of signal communication taking place in homogeneous soil medium, the path loss during this case is essentially lower. But, for the signal transmission, this effect is entirely influenced on the MI, which may be a single parameter which will be taken into consideration for optimization and network architecture. Generally, it is hard to improve the position and even the orientation of nodes, because the distribution of nodes is typically attainable to some certain locations and under a particular angle. But, if this degree of freedom is accessible to the system engineer, specific improvement generally causes encouraging results and would be used for the improvement in the overall performance of the network.

4.5. Cross-Layer Improvement. The sensor network capacity or throughput for wireless sensor networks has been described in detail in [99]. Further, for MI-based WUSNs, authors in [80] discussed and measured the network performance for these networks, using the channel model as explained in [9]. Interestingly, as compared to conventional EM-based communication networks, the performance was observed to be degraded in the case of MI-WUSNs, but in the study made in [80], general issues of the signal process and scheduling were not being taken under consideration. Therefore, some advanced designs of MI-WUSNs with multiple sensors interacting in the improved cross-layered model are refined, e.g., [100].

This work is taken into consideration using various dimensions of signal processing and networking, e.g., modulation techniques, linking methodologies, routing mechanisms, scheduling, error rates, and distribution techniques. Similarly, different sets of system parameters that rely upon the presumed channel models and surroundings are recommended. Particularly, various distribution readying options like direct MI communication using no passive relays and MI waveguides using passive relays are analyzed under realistic architecture checks in [101]. More related work [102] provides some vision into the network architecture majorly for the medium access control (MAC) layer. The evolution from traditional protocol architecture to cross-layered protocol architecture for MI-WUSNs is discussed in many studies.

Additionally, a SWIPT design for the underground communication is covered in [103], considering the power consumption and battery charging requirements for the network architecture. The method assigns unique priorities to the sensor nodes as per the remaining battery life and thereby slightly changing the definition of throughput of sensor networks. However, this is a very fruitful and promising method because it increases the exploitation network time and reduces the chances of disconnection caused by the alleged energy holes [104] that arise if a number of the foremost drained nodes dry up by battery power.

Nguyen et al. [105] proposed an idea of a hybrid clustering and evolutionary approach for WUSN's lifetime maximization [106]. The advantages and disadvantages for the cross-layered approach have been presented in Table 1.

4.6. Sensor Battery Charging. According to [3], the battery has been found to be another crucial concern for any WSN. In recent applications of WSNs, the charging of all the sensing nodes using a movable charging vehicle having a better charging framework and a moving path is used [107]. The same mechanism may be applied to MIWUSNs as well with minor alterations attributing to characteristics of underground medium as well architecture of transceiver nodes based on the system model. The popular strategies of energy linking [108] are used, but their performance remains unpredictable for underground medium. Additionally, in many WUSN applications, due to sensor 
TABLE 1: Advantages and disadvantages for the crossed-layered approach.

Crossed-layered approach

Advantages

(a) Cross-layered approach improves the quality of service in mobile networks

(b) It helps in the reduction of the congestion in various wireless communication applications

(c) It is used in various routing mechanisms

(d) It is helpful in efficiently exchanging multimedia resources over the wireless networks

(e) It supports information exchange and optimization across different layers

(f) Easier to optimize, maintain, and manage protocols

(g) Simplifying the design by breaking the system by end-to-end networking

(h) It provides clear interfaces and modular architecture

Disadvantages

(a) Cross-layered approach suffers from lack of proper architecture

(b) Uncontrolled cross-layer patterns may cause chaos

(c) Unforeseen dependencies may be activated among layers due to unexpected cross-layer interaction

(d) It is not easy to integrate cross-layer designs

(e) More chances of stability concerns are there, if not addressed properly

(f) Information of operation at one layer cannot be used at another

(g) Introduces inefficiencies and redundancies

(h) Redundant functionality leads to data overhead and processing

nodes being distributed in rarely reachable environments such as oil reservoirs and mines $[76,109]$ and infrastructural health observation [10], a moving agent might not be capable enough to come close to each sensing node for charging purpose. In addition, electromagnetism waves might not be capable of penetrating the surface to approach the sensing nodes for transferring power. When a number of the sensing devices are reached by the charging vehicle, its energy consumption might be one of the essential parameters for comprehensive analysis of the costs.

As an alternative, a fixed power supply placed above the surface of ground and connected with other sensing devices, e.g., via cable, is employed. The complete network is then wirelessly charged with the help of this charged node $[59,103]$. The improvement of the corresponding charging procedure is explained in [109], where energy losses are minimized due to a virtual magnetic MIMO-based relay network associated with the optimized transmission protocol. Due to some limitations, the charging capability remains very low, while coils with moderate radius (approx. $25 \mathrm{~cm}$ ) were used, although energy capability may be increased by improving the system parameters as part of the mentioned cross-layer environment.

Moreover, extra passive relays [97] can be placed and even organized in MI waveguides. In such situations, because of the increased spatial diversity of the communication system, the positioning of relays seems acceptable, which is extremely helpful, particularly when coupled with the aforementioned virtual MIMO approach. Additionally, relay devices are generally used, which consume negligible energy while transmitting the information but still support the energy relaying at that time. It is very useful that these relay devices are generally presumed to be most of the time fully charged needing no energy for getting themselves charged. This is because the relay devices would not take part in information transmission; it is not connected with any network link. Therefore, they are often deployed among the device nodes, in a way that the distance between the relaying devices and any neighboring sensor nodes is as minimum as possible. Similarly, it becomes possible to improve the charging capability and decrease the path loss upper limit caused by energy relaying. The charging capability would further improve, if numbers of relays are increased.

4.7. Localization. The different aspects of localization [110] have been studied well for MI-based networks in some previous works, e.g., [111, 112]. Nevertheless, these works do not effectively acknowledge the localization in underground medium having considerably distinct properties and challenges. Therefore, the strategies suggested in these works are not fit for MI-based WUSNs.

The major application domains of the localization within the underground medium are pursuit of natural wildlife [113] and location of humans and robots in mines [114]. The researchers in [113] have described the utilization of MI transceivers for the purpose of confining small rabbits and badgers in their natural environment. Still, localization and pursuit in [113] have not powered immediate detection, yet on an eternal data gathering. Additionally, an excellent scheme is suggested in [113], which applies long coils placed aboveground. In spite of significantly accurate localization illustrated in this work, the suggested technique is simply appropriate for the localization in limited sized areas, because it requires much placement efforts. The study of the localizing humans trapped in mines by using various signal transmission schemes such as ZigBee and RFID for detection has been provided in [115]. However, in the event of potential cave-ins, a portable magnetic sensor put on the body of a mine worker is also employed to mark the location 
TABLE 2: SWOT analysis of EM/MI-WUSNs.

Strengths

(1) The medium transfer from one device to another is optimized and reduces the number of downloads and size

(2) The devices can send and receive messages in real time

(3) Each device receives media and message only from the device associated with him

(4) Economically feasible: low cost as compared to other products available in markets

(5) Incorporation of a new device is easy

Weaknesses

(1) Processes are slow as compared to wired networks

(2) Limited battery power and storage

(3) Each new device requires certification before installing

Opportunities

(1) Open-source hardware

(2) Technological advancement

(3) Innovation

(4) New business field for the tycoons

Threats

(1) Insecure network

(2) Not being aware of the environment

(3) Regulatory issues by statutory governing bodies

(4) Awarelessness

(5) High humidity and temperature can cause the damage or malfunctioning to the devices

of the miners. For these cases, the idea of applied machine learning-based localization has been proposed in [101] to notify the nonlinearity of the transmission channel that forces the lowering of localization accuracy with conventional localization strategies like trilateration or triangulation. The authors of [8] advised a unique gradient-based technique and a localization framework for localization in tunnels. After analyzing the path loss of EM waves propagating in mines, MI-WUSNs have been applied for the safety of the miners [114].

The ambiguity in the received signal due to a potential coil misalignment, positioning errors, etc. is the unaddressed issues in the localization dependent on the MI. The positions of the sensors could be extensively changed, particularly in the event of a mine collapse. The effect on quality of localization due to this divergence from the assumed positions remains an unanswered challenge for the future.

4.8. Experimental Work in MI-WUSNs. In addition to putting theoretical system models, a large experimental work has also been carried out by researchers to prove the models along with assumptions [116]. These validations have partly approved different dimensions of the signal communication in MI-WUSNs, e.g., the signals getting exhausted due to soil conductivity (eddy currents) and signals getting filtered within MI relays. The experiments have also been performed using test beds in individual laboratories for, e.g., [117].

Specifically, a number of (at least seven) passive relays arranged on a straight line with the complete communication range of a few meters have been examined in the MI waveguide framework [94].

At different frequencies, the mean received signal power is recorded to be in range of 4 to $9 \mathrm{MHz}$. Distinct environ- mental factors attributing to unnatural rise of water content in soil have also been evaluated. Further, a 3D magnetic coil is placed; also, corresponding changes in performance are observed. In [117], a test bed with multiple sensor nodes is enforced, which uses 3D coils for the purpose of spatial multiplexing. The transmission range of 35 meters has been illustrated using nodes having applied career frequency of $2 \mathrm{kHz}$, establishing that coil orientation has nothing to do with received signal power.

In another experiments, for, e.g., [116], the transceivers are placed in soil in the field. In [116], the signal attenuation is calculated to validate the result of eddy currents in soil, causing the increase in path loss of signals. Here, an explicit MI link with no passive relays has been thought about for communication ranges up to thirty meters having the operational frequency of $5 \mathrm{kHz}$. Overall, experimental results have been found in accordance with signal transmission models put theoretically. These results also indicate that the extra attention incorporated by the theoretical works is correct.

However, due to high system complexity and deployment efforts, no field experiments have yet been described or done on completely operative multinode MI-WUSNs having communication with minimal past loss, efficient scheduling, and charging as well as localization. However, the chances of developing protocols and field experiments are on an increasing trend due to high interest of researchers in this area.

\section{Analysis of WUSN}

5.1. SWOT Analysis of EM/MI-WUSN. The SWOT analysis of EM/MI communication-based WUSNs helps in analyzing the key points leading to performance improvement. The 
TABLE 3: Comparative study of WSN protocols.

\begin{tabular}{|c|c|c|c|c|c|}
\hline $\begin{array}{l}\text { Sr. } \\
\text { no. }\end{array}$ & Protocol & $\begin{array}{l}\text { Performance } \\
\text { parameters }\end{array}$ & $\begin{array}{c}\text { Energy } \\
\text { consumption }\end{array}$ & Benefits & Limitations \\
\hline 1. & $\begin{array}{l}\text { Underground } \\
\text { opportunistic routing } \\
\text { (UnOR) protocol }\end{array}$ & $\begin{array}{l}\text { Throughput ratio, } \\
\text { energy } \\
\text { consumption }\end{array}$ & High & $\begin{array}{l}\text { Buffer data through overhearing, } \\
\text { high link quality }\end{array}$ & High energy consumption \\
\hline 2. & $\begin{array}{l}\text { Cross-layer protocol } \\
\text { (XLP) }\end{array}$ & $\begin{array}{c}\text { Energy } \\
\text { consumption, } \\
\text { route failure rate }\end{array}$ & Moderate & $\begin{array}{l}\text { Improved communication } \\
\text { performance }\end{array}$ & High operation overhead \\
\hline 3. & $\begin{array}{c}\text { Mobile node-based } \\
\text { routing }(\mathrm{MNBR})\end{array}$ & $\begin{array}{c}\text { Energy } \\
\text { consumption }\end{array}$ & High & $\begin{array}{l}\text { Quick path routing, balanced use } \\
\text { of node energy }\end{array}$ & Involve enormous overhead \\
\hline 4. & $\begin{array}{l}\text { Unicast-based gradient } \\
\text { routing protocol } \\
(\mathrm{UGRP})\end{array}$ & $\begin{array}{l}\text { Network lifetime, } \\
\text { packet delivery } \\
\text { ratio }\end{array}$ & Moderate & Less redundant transmission & Energy consumption \\
\hline 5. & $\begin{array}{l}\text { Energized routing and } \\
\text { multicast tree-based } \\
\text { geocasting protocol } \\
\text { (ERMTG) }\end{array}$ & $\begin{array}{l}\text { Packet delivery } \\
\text { ratio, path energy, } \\
\text { network energy }\end{array}$ & Moderate & $\begin{array}{l}\text { Better delivery ratio, lower energy } \\
\text { consumption }\end{array}$ & More computational overhead \\
\hline 6. & $\begin{array}{l}\text { Efficient data multiple } \\
\text { aggregation (EDMA) }\end{array}$ & $\begin{array}{c}\text { Data loss, data sent } \\
\text { ratio }\end{array}$ & Moderate & $\begin{array}{c}\text { Minimized packet loss, data } \\
\text { redundancy }\end{array}$ & $\begin{array}{c}\text { Similarity function is not } \\
\text { satisfactory }\end{array}$ \\
\hline 7. & $\begin{array}{l}\text { Round-based clustering } \\
\text { (RBC) }\end{array}$ & $\begin{array}{c}\text { Energy } \\
\text { consumption, } \\
\text { throughput, data } \\
\text { received ratio }\end{array}$ & Low & $\begin{array}{l}\text { Less energy consumption, high } \\
\text { throughput }\end{array}$ & Node mobility problem \\
\hline 8. & $\begin{array}{l}\text { Distributed } \\
\text { environment-aware } \\
\text { protocol (DEAP) }\end{array}$ & $\begin{array}{l}\text { Security, } \\
\text { throughput gain }\end{array}$ & Low & $\begin{array}{l}\text { More secure, operate on separate } \\
\text { layers energy saving, high } \\
\text { throughput gain, low } \\
\text { computational complexity }\end{array}$ & Statistical delay \\
\hline 9. & $\begin{array}{l}K \text {-means and analysis } \\
\text { of variance (ANOVA) }\end{array}$ & $\begin{array}{c}\text { Energy } \\
\text { consumption, data } \\
\text { sent ratio } \\
\end{array}$ & Moderate & Minimized data redundancy & Higher energy depletion \\
\hline 10. & $\begin{array}{l}\text { Scalable and efficient } \\
\text { data gathering routing } \\
\text { protocol (SEDG) }\end{array}$ & $\begin{array}{l}\text { Delay, energy } \\
\text { consumption, } \\
\text { network } \\
\text { throughput }\end{array}$ & Low & $\begin{array}{l}\text { Increased network lifetime, } \\
\text { improved packet delivery ration }\end{array}$ & Data collection efficiency issue \\
\hline 11. & $\begin{array}{l}\text { Path reliability-aware } \\
\text { data delivery protocol } \\
\text { (PRADD) }\end{array}$ & $\begin{array}{l}\text { Packet delivery } \\
\text { ratio, routing } \\
\text { overhead, energy } \\
\text { consumption }\end{array}$ & Low & $\begin{array}{l}\text { Lower overhead, energy } \\
\text { consumption }\end{array}$ & Lack of dynamic data transfer \\
\hline 12. & $\begin{array}{l}\text { Delay-tolerant mobile } \\
\text { sink model-nonzero } \\
\text { (DTMSM-NZ) }\end{array}$ & $\begin{array}{l}\text { Packet delivery } \\
\text { ratio, latency, } \\
\text { throughput ratio }\end{array}$ & Moderate & Lowest mobile sink travel time & Involve enormous overhead \\
\hline 13. & $\begin{array}{l}\text { Multihop graph-based } \\
\text { approach for an energy- } \\
\text { efficient routing (MH- } \\
\text { GEER) }\end{array}$ & $\begin{array}{l}\text { Residual energy of } \\
\text { distant } \mathrm{CH} \text { nodes, } \\
\text { load balancing }\end{array}$ & Low & $\begin{array}{l}\text { ACO-based algorithm to achieve } \\
\text { multihop routing, better load } \\
\text { balancing }\end{array}$ & $\begin{array}{c}\text { Lack of congestion awareness } \\
\text { mechanism }\end{array}$ \\
\hline 14. & $\begin{array}{l}\text { Low-energy adaptive } \\
\text { clustering hierarchy } \\
\text { protocol (LEACH) }\end{array}$ & $\begin{array}{c}\text { Energy } \\
\text { consumption }\end{array}$ & Moderate & $\begin{array}{l}\text { Energy-efficient and cluster-based } \\
\text { hierarchical and efficient routing } \\
\text { distributes the load equally }\end{array}$ & $\begin{array}{l}\text { Data aggregation is carried out at } \\
\text { the cluster head level, single-hop } \\
\text { communication, load is not equally } \\
\text { distributed }\end{array}$ \\
\hline 15. & $\begin{array}{c}\text { Energy-aware multihop } \\
\text { multipath hierarchical } \\
\text { (EAMMH) }\end{array}$ & $\begin{array}{l}\text { No. of dead nodes, } \\
\text { average energy }\end{array}$ & Low & $\begin{array}{c}\text { Better average energy, multipath } \\
\text { hierarchical routing }\end{array}$ & - \\
\hline
\end{tabular}

strengths indicate the key areas of WUSN, where it can be used in an efficient manner, and weaknesses indicate the key points to be focused for the research to overcome the issues.
The opportunities indicate the direction of future advancements, and threats have to be taken care of while implementing WUSNs. The SWOT analysis is given in Table 2. 


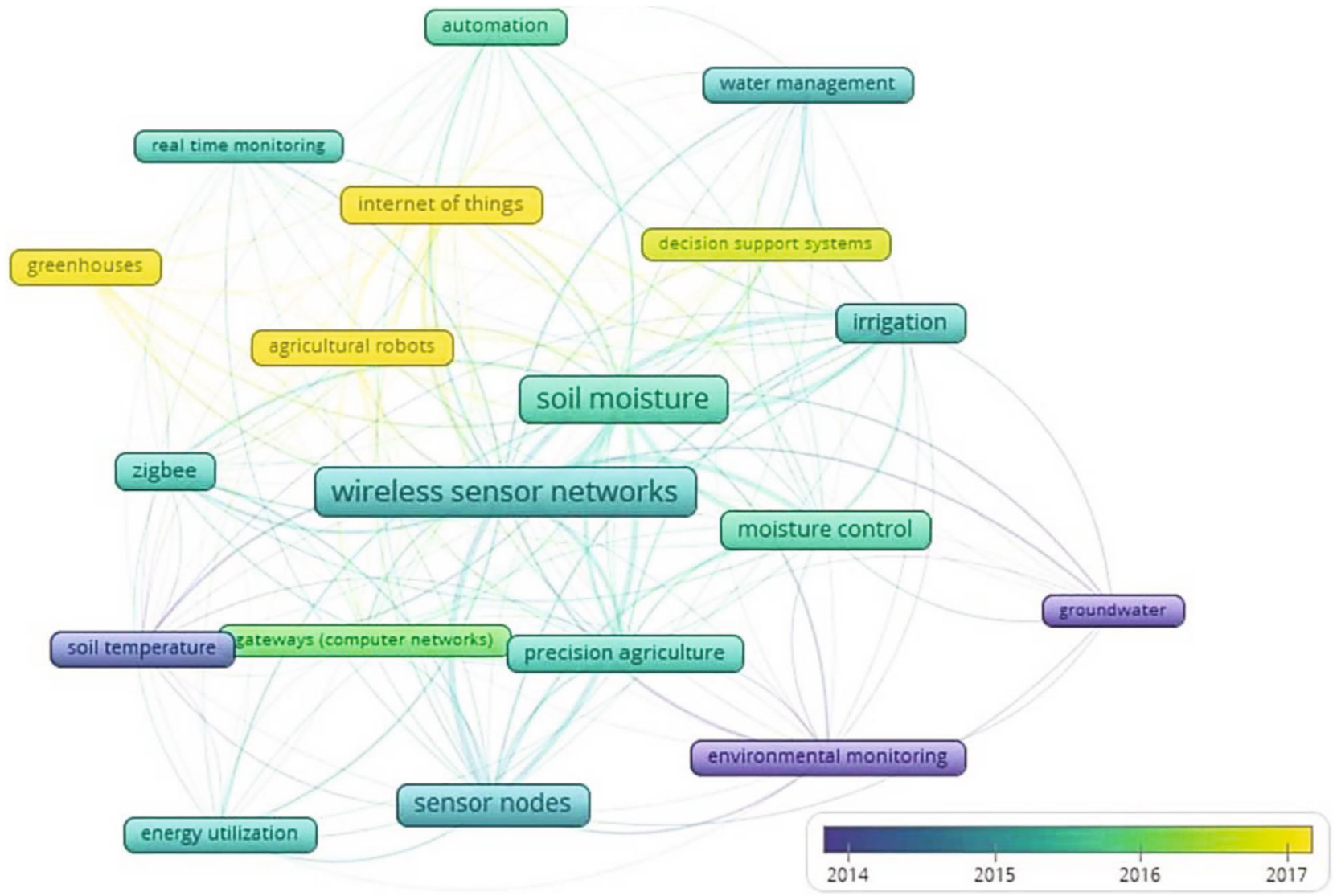

FIGURE 3: Scientometric analysis of WSN and soil performed using VOSviewer.

5.2. Comparative Study of Protocols. The protocols used in WSN technologies such as underground and underwater WSNs and cross-layered architecture using EM/MI communication are considered for the comparative study. This study contains major protocols starting from year 2009 till date with their performance parameters, benefits, and limitations. This study considers the major developments in protocols used in WSNs which indicate the directions of research in WSN. Table 3 represents the comparative study of WSN protocols.

For energy-constrained underground environment for WUSNs, conventional OSI layered protocols are more expensive and therefore are less preferred from perspective of effectiveness and efficiency of MI-WUSNs. Rather crosslayered protocols prove to be better for the same, where interfacing of functionalities of different layers may be utilized in an integrated manner. Out of the above said protocols, the distributed environment-aware protocol (DEAP) is more secure and operates on separate layers leading to energy saving, high-throughput gain, and low computational complexity.

5.3. Enabling Technologies. Many recent developments in UWSNs have seen tremendous growth in various areas. Technological communication developments permit interaction between underwater sensors and applications to deliver user services and were adjusted to different requirements and preferences. The breakthrough in Industry 4.0 has become a motive factor for the adoption of Industry IoT (IIoT). IIoT offers secure data transmission and update from time to time to the cloud network from different sensors. IIoT is a single system, consisting of cloud networks, terminals, equipment, and machines, to incorporate industrial wireless networks and IoT technology [118]. Recent advancements in the IoT and UWSN industry have therefore led to a growing interest in the Internet of Underwater Things (IoUT). IoUT enables a number of underwater sensors to interconnect by gathering and transferring highspeed data to the surface station.

A notable further step forward in reducing the harmful effects of greenhouse gas on the environment is the introduction of a green IoUT [119]. In addition to this greenhouse effect, authors state that underwater nodes and vehicles demand significant power consumption that may halt vital tasks or applications from operating prematurely. This issue has led researchers in developing emerging energy-efficient UWSN designs for offshore gas and petroleum environments in particular [120]. Due to the integration of heterogeneous nodes, underwater communication systems, complicated architecture, and various underwater applications, underwater communications systems will face challenges during the next few years. Authors have therefore proposed a software-defined network (SDN) paradigm that promotes practices of innovation, improves the flexibility of the network, efficiently assigns resources, and manages operating costs [121]. 


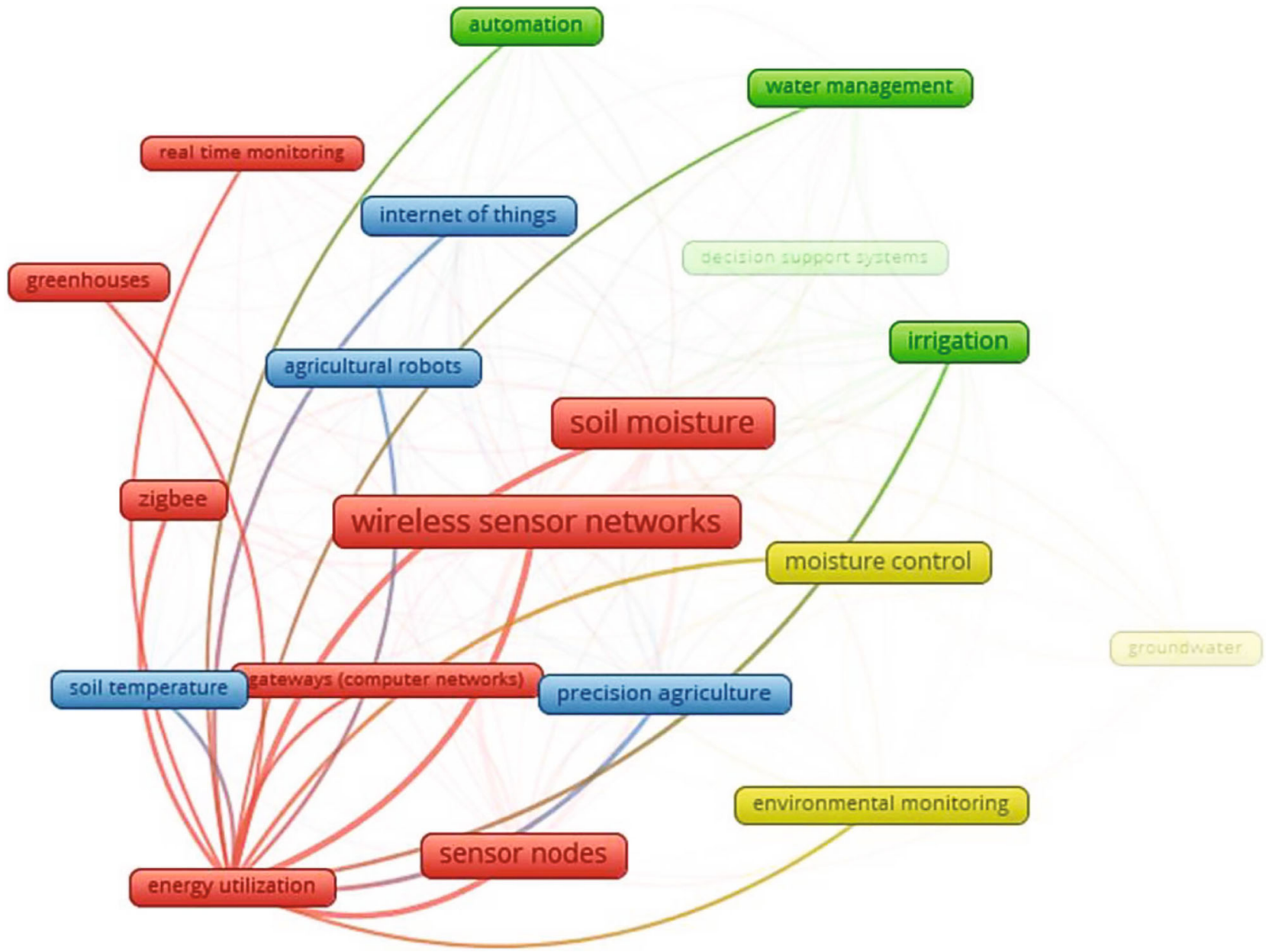

Figure 4: Network analysis of the relationship between "energy utilization" with other domains.

\section{Inference and Further Challenges of MI- WUSNs}

The recent advancement in MI-WUSNs has shifted the prime research scope in this field to a number of new challenges which are still to be addressed and resolved.

One such important area of MI-WUSN evolution is to design the different interfacing mechanisms between MIbased WUSNs and other categories of WSNs such as WUSNs interfacing with wireless underwater sensor networks (as in the case of exploration of deep ocean and naval border defense), WUSNs interfacing with WSNs for plantations like trees and mushrooms, WUSNs interacting with WSNs for livestock (as in the case of a detailed study of animal lives and their effect on environment), WUSNs having an interface with the power grid (as in the case of monitoring structural health), or WUSNs communicating with self-driving cars (as in the case of navigation and charging). The possible issues likely to come in planning of these interfaces are yet to be looked into in forthcoming days.

The design of the MI waveguide network having combined usage of active and passive relaying is also one challenging area which however offers joint benefits of low path loss as the intermediate frequency as well as minimized frequency level but at the same time is extremely difficult to design because it is important to determine the appropriate location and operation pattern for each relay.

One additional way to further boost signal quality and other output parameters in MI-WUSNs is to deploy multiple orthogonal coils for each transceiver node. Despite the unintended misalignment of a number of nodes, this approach guarantees a specific connectivity degree but is quite challenging to be designed.

One very major factor generally underestimated in regard to system performance is imperfect channel state information (CSI). Generally, any system parameter getting deviated might lead to the entire theoretical model as well as network architecture to be invalid. Therefore, the majority of the presently available solutions should be upgraded, incorporating robust updation and adjustment. Some of the instances are connecting the mobile surface system to a network for backhauling or varying soil wetness resulting in the additional critical modification of the channel state (e.g., during rainfalls) and a network delaying its charging in the case of high power loss within the medium. In general, the system performance is maximized for all appropriate channel states by introducing various types of operation mechanisms (e.g., collecting data, localization, and charging) with separate sets of initially optimized system parameters that can be taken according to the channel state and function. 
While various authors typically suggest cross-layer optimization, but a multiobjective optimization, that optimizes system efficiency in terms of charging capability, accuracy of localization and network throughout still remains a challenging issue. This multiobjective optimization, particularly the design of self-charging and power-efficient networks under the constraint of a highly viable performance and a low valued maximum localization error, seems very promising. It can be attained by setting various system parameter sets that can be selected as per operational mode and application. For example, higher frequency may be used for short communication links (network and charging mode) and lower frequency may be used for locating distant target nodes (localization mode). Similarly, in order to achieve efficiency that would be similar to optimal in most situations, the network could switch into the most acceptable mode.

The scientometric analysis has been performed using the VOSviewer analytical tool to investigate recent developments in the area of WSN and soil monitoring. The database used for analysis has been extracted from Scopus for the last six years. The network of keywords has been displayed in Figure 3 which indicates the extensive implementation of WSN technologies in soil, moisture control, and precision agriculture. The implementation of automation, Internet of things, and sensor nodes are also studied. In last two years, the research on WSN with applications on Internet of things, decision support systems, greenhouses, and agriculture robots has been reported.

Figure 4 plots the network analysis of energy utilization with various domains studied related to WSN. The relationship between "energy utilization" and decision support systems and groundwater has not been studied. On the other hand, moisture control and environmental monitoring are recently explored by the researchers which have plenty of scope of research. It can be deduced from the present scientometric investigation that energy utilization through WSN and other allied domains can be further explored. Energy is a key source of expenditure in agriculture in India which can be efficiently managed through implementation of WSN, Internet of things, automation, and decision support systems.

\section{Conclusion}

Underground WSNs being a special class of WSNs cater to a large range of application areas making it an attractive destination area for researchers. This review paper has highlighted the recent developments happened in areas closely associated with MI-WUSNs such as EM-based WUSNs, underwater sensor networks, and MI-based signal transmission mechanisms such as NFC and WPT. The advancements in these areas present a vital picture about possible concerns and their redressal mechanisms related to system modelling and design, which prove to be beneficial for designing efficient MI-WUSNs. The developments made in multiple dimensions of MI-WUSNs ranging from deployment methodologies, channel modelling, synchronization and channel estimation, single MI links, localization capacity, and battery charging requirements to cross-layered architecture to attain high quality of service, have been discussed. These factors have led the emphasis of the research to new horizons. A large scope is there to work for more efficient and optimized cross-layered techniques, which could integrate multiple MI-WUSN functionalities, such as low energy requirement but high network throughput. It would also be promising to use combination of active and passive MI relay devices operating differently reducing the overall path loss and energy requirements. Various interfacing possibilities of WSNs with underground WSNs need to be exploited including self-driving vehicles and other IoT applications.

\section{Conflicts of Interest}

The authors declare no conflict of interest.

\section{References}

[1] I. F. Akyildiz and E. P. Stuntebeck, "Wireless underground sensor networks: research challenges," Ad Hoc Networks, vol. 4, no. 6, pp. 669-686, 2006.

[2] S. Kisseleff, I. F. Akyildiz, and W. H. Gerstacker, "Survey on advances in magnetic induction-based wireless underground sensor networks," IEEE Internet of Things Journal, vol. 5, no. 6, pp. 4843-4856, 2018.

[3] I. F. Akyildiz, W. Su, Y. Sankarasubramaniam, and E. Cayirci, "Wireless sensor networks: a survey," Computer Networks, vol. 38, no. 4, pp. 393-422, 2002.

[4] I. F. Akyildiz and M. C. Vuran, Wireless sensor networks, volume 4, John Wiley \& Sons, 2010.

[5] H. Huang, J. Shi, F. Wang, D. Zhang, and D. Zhang, "Theoretical and experimental studies on the signal propagation in soil for wireless underground sensor networks," Sensors, vol. 20 , no. 9, p. $2580,2020$.

[6] M. Can Vuran and A. R. Silva, "Communication through soil in wireless underground sensor networks-theory and practice," in Sensor Networks, pp. 309-347, Springer, 2010.

[7] R. Bansal, "Near-field magnetic communication," IEEE Antennas and Propagation Magazine, vol. 46, no. 2, pp. 114-115, 2004.

[8] S.-C. Lin, A. A. Alshehri, P. Wang, and I. F. Akyildiz, "Magnetic induction-based localization in randomly deployed wireless underground sensor networks," IEEE Internet of Things Journal, vol. 4, no. 5, pp. 1454-1465, 2017.

[9] Z. Sun and I. F. Akyildiz, "Magnetic induction communications for wireless underground sensor networks," IEEE transactions on antennas and propagation, vol. 58, no. 7, pp. 2426-2435, 2010.

[10] C. Park, Q. Xie, P. H. Chou, and M. Shinozuka, "Duranode: wireless networked sensor for structural health monitoring," SENSORS, vol. 2005, 2005.

[11] P. Aleotti and R. Chowdhury, "Landslide hazard assessment: summary review and new perspectives," Bulletin of Engineering Geology and the Environment, vol. 58, no. 1, pp. 21-44, 1999.

[12] X. Tan and Z. Sun, "An optimal leakage detection strategy for underground pipelines using magnetic induction-based sensor networks," in International Conference on Wireless Algorithms, Systems, and Applications, pp. 414-425, Springer, 2013. 
[13] S. Basu, S. Pramanik, S. Dey, G. Panigrahi, and D. K. Jana, "Fire monitoring in coal mines using wireless underground sensor network and interval type-2 fuzzy logic controller," International Journal of Coal Science \& Technology, vol. 6, no. 2, pp. 274-285, 2019.

[14] S. Wu, A. C. M. Austin, A. Ivoghlian, A. Bisht, and K. I.-K. Wang, "Long range wide area network for agricultural wireless underground sensor networks," Journal of Ambient Intelligence and Humanized Computing, pp. 1-17, 2020.

[15] D. Wohwe Sambo, A. Forster, B. O. Yenke, I. Sarr, B. Gueye, and P. Dayang, "Wireless underground sensor networks path loss model for precision agriculture (WUSM-PLM)," IEEE Sensors Journal, vol. 20, no. 10, pp. 5298-5313, 2020.

[16] A. Sheth, K. Tejaswi, P. Mehta et al., "Senslide: a sensor network based landslide prediction system," in Proceedings of the 3rd international conference on embedded networked sensor systems, pp. 280-281, San Diego, California, USA, 2005.

[17] K. Martinez, R. Ong, and J. Hart, "Glacsweb: a sensor network for hostile environments," in 2004 First Annual IEEE Communications Society Conference on Sensor and Ad Hoc Communications and Networks, pp. 81-87, Santa Clara, CA, USA, 2004.

[18] G. Werner-Allen, K. Lorincz, M. Ruiz et al., "Deploying a wireless sensor network on an active volcano," IEEE Internet Computing, vol. 10, no. 2, pp. 18-25, 2006.

[19] G.-S. Kim, Y.-S. Son, J.-H. Lee et al., “Air pollution monitoring and control system for subway stations using environmental sensors," Journal of Sensors, vol. 2016, Article ID 1865614, 10 pages, 2016.

[20] F. K. Banaseka, H. Franklin, F. A. Katsriku, J.-D. Abdulai, A. Ekpezu, and I. Wiafe, "Soil medium electromagnetic scattering model for the study of wireless underground sensor networks," Wireless Communications and Mobile Computing, vol. 2021, Article ID 8842508, 11 pages, 2021.

[21] D. G. Dudley, M. Lienard, S. F. Mahmoud, and P. Degauque, "Wireless propagation in tunnels," IEEE Antennas and Propagation Magazine, vol. 49, no. 2, pp. 11-26, 2007.

[22] M. C. Vuran and I. F. Akyildiz, "Channel model and analysis for wireless underground sensor networks in soil medium," Physical communication, vol. 3, no. 4, pp. 245254, 2010.

[23] M. C. Vuran and I. F. Akyildiz, "Xlp: a cross-layer protocol for efficient communication in wireless sensor networks," IEEE Transactions on Mobile Computing, vol. 9, no. 11, pp. 1578-1591, 2010.

[24] A. R. Silva and M. C. Vuran, "Empirical evaluation of wireless underground-to-underground communication in wireless underground sensor networks," in International Conference on Distributed Computing in Sensor Systems, pp. 231-244, Springer, Marina del Rey, CA, USA, 2009.

[25] H. Zemmour, G. Baudoin, and A. Diet, "Soil effects on the underground-to- aboveground communication link in ultrawideband wireless underground sensor networks," IEEE Antennas and Wireless Propagation Letters, vol. 16, pp. 218-221, 2017.

[26] A. R. Silva and M. C. Vuran, “(CPS)^2: integration of center pivot systems with wireless underground sensor networks for autonomous precision agriculture," in Proceedings of the 1st ACM/IEEE international conference on cyber-physical systemspp. 79-88, Stockholm, Sweden, 2010.
[27] X. Yu, W. Han, and Z. Zhang, "Path loss estimation for wireless underground sensor network in agricultural application," Agricultural Research, vol. 6, no. 1, pp. 97-102, 2017.

[28] A. Salam and M. C. Vuran, "Impacts of soil type and moisture on the capacity of multi-carrier modulation in Internet of underground things," in 2016 25th International Conference on Computer Communication and Networks (ICCCN), pp. 1-9, Waikoloa, Hawaii, USA, 2016.

[29] G. Liu, Z. Wang, and T. Jiang, "QoS-aware throughput maximization in wireless powered underground sensor networks," IEEE Transactions on Communications, vol. 64, no. 11, pp. 4776-4789, 2016.

[30] B. Yuan, H. Chen, and X. Yao, "Optimal relay placement for lifetime maximization in wireless underground sensor networks," Information Sciences, vol. 418-419, pp. 463-479, 2017.

[31] A. Salam and M. C. Vuran, "Smart underground antenna arrays: a soil moisture adaptive beamforming approach," in IEEE INFOCOM 2017-IEEE Conference on Computer Communications, pp. 1-9, Atlanta, GA, USA, 2017.

[32] A. Salam, M. C. Vuran, and S. Irmak, "Di-sense: in situ realtime permittivity estimation and soil moisture sensing using wireless underground communications," Computer Networks, vol. 151, pp. 31-41, 2019.

[33] N. T. Tam, D. A. Dung, T. H. Hung, H. T. T. Binh, and S. Yu, "Exploiting relay nodes for maximizing wireless underground sensor network lifetime," Applied Intelligence, vol. 50, no. 12 , pp. $4568-4585,2020$.

[34] H. T. H. Trang, L. T. Dung, and S. O. Hwang, "Connectivity analysis of underground sensors in wireless underground sensor networks," Ad Hoc Networks, vol. 71, pp. 104-116, 2018.

[35] A. E. Forooshani, S. Bashir, D. G. Michelson, and S. Noghanian, "A survey of wireless communications and propagation modeling in underground mines," IEEE Communications surveys \& tutorials, vol. 15, no. 4, pp. 1524-1545, 2013.

[36] X. Dong, M. C. Vuran, and S. Irmak, “Autonomous precision agriculture through integration of wireless underground sensor networks with center pivot irrigation systems," Ad Hoc Networks, vol. 11, no. 7, pp. 1975-1987, 2013.

[37] K. Finkenzeller, RFID Handbook: Fundamentals and Applications in Contactless Smart Cards, Radio Frequency Identification and Near-Field Communication, John wiley \& sons, 2010.

[38] J. J. Sojdehei, P. N. Wrathall, and D. F. Dinn, "Magnetoinductive (mi) communications," in MTS/IEEE Oceans 2001. An Ocean Odyssey. Conference Proceedings (IEEE Cat. No. 01CH37295), vol. 1, pp. 513-519, Honolulu, HI, USA, 2001.

[39] G. Madlmayr, J. Langer, C. Kantner, and J. Scharinger, "NFC devices: security and privacy," in 2008 Third International Conference on Availability, Reliability and Security, pp. 642647, Barcelona, Spain, 2008.

[40] L. R. Varshney, P. Grover, and A. Sahai, "Securing inductively-coupled communication," in 2012 Information theory and applications workshop, pp. 47-53, San Diego, CA, USA, 2012.

[41] H. C. Jing and Y. E. Wang, "Capacity performance of an inductively coupled near field communication system," in 2008 IEEE Antennas and Propagation Society International Symposium, pp. 1-4, San Diego, CA, USA, 2008. 
[42] G. Dumphart and A. Wittneben, "Stochastic misalignment model for magneto-inductive SISO and MIMO links," in 2016 IEEE 27th Annual International Symposium on Personal, Indoor, and Mobile Radio Communications (PIMRC), pp. 1-6, Valencia, Spain, 2016.

[43] H. Guo and Z. Sun, "Increasing the capacity of magnetic induction communication using MIMO coil-array," in 2016 IEEE Global Communications Conference (GLOBECOM), pp. 1-6, Washington DC, USA, 2016.

[44] H. Nguyen, J. I. Agbinya, and J. Devlin, "FPGA-based implementation of multiple modes in near field inductive communication using frequency splitting and MIMO configuration," IEEE Trans- actions on Circuits and Systems I: Regular Papers, vol. 62, no. 1, pp. 302-310, 2014.

[45] M. Masihpour and J. I. Agbinya, "Cooperative relay in near field magnetic induction: a new technology for embedded medical communication systems," in 2010 Fifth International Conference on Broadband and Biomedical Communications, pp. 1-6, Malaga, Spain, 2010.

[46] M. Masihpour, D. Franklin, and M. Abolhasan, "Multihop relay techniques for communication range extension in near-field magnetic induction communication systems," Journal of Networks, vol. 8, no. 5, 2013.

[47] J. I. Agbinya, Principles of Inductive Near Field Communications for Internet of Things, vol. 18, River Publishers, 2011.

[48] B. Gulbahar, "Network topology modulation for energy and data transmission in Internet of magneto-inductive things," in 2016 IEEE Globecom Workshops (GC Wkshps), pp. 1-6, Washington DC, USA, 2016.

[49] N. Shinohara, Wireless power transfer via radiowaves, Wiley Online Library, 2013.

[50] K. Fotopoulou and B. W. Flynn, "Wireless power transfer in loosely coupled links: coil misalignment model," IEEE Transactions on Magnetics, vol. 47, no. 2, pp. 416-430, 2010.

[51] A. Kurs, A. Karalis, R. Moffatt, J. D. Joannopoulos, P. Fisher, and M. Soljačić, "Wireless power transfer via strongly coupled magnetic resonances," science, vol. 317 , no. 5834, pp. 83-86, 2007.

[52] A. P. Sample, D. T. Meyer, and J. R. Smith, "Analysis, experimental results, and range adaptation of magnetically coupled resonators for wireless power transfer," IEEE Transactions on Industrial Electronics, vol. 58, no. 2, pp. 544-554, 2011.

[53] Y. Zhang, Z. Zhao, and K. Chen, "Frequency splitting analysis of magnetically- coupled resonant wireless power transfer," in 2013 IEEE Energy Conversion Congress and Exposition, pp. 2227-2232, Denver, Colorado, USA, 2013.

[54] B. W. Flynn and K. Fotopoulou, "Rectifying loose coils: wireless power transfer in loosely coupled inductive links with lateral and angular misalignment," IEEE Microwave Magazine, vol. 14, no. 2, pp. 48-54, 2013.

[55] J. Park, Y. Tak, Y. Kim, Y. Kim, and S. Nam, "Investigation of adaptive matching methods for near-field wireless power transfer," IEEE Transactions on Antennas and Propagation, vol. 59, no. 5, pp. 1769-1773, 2011.

[56] S. Lukic and Z. Pantic, "Cutting the cord: static and dynamic inductive wireless charging of electric vehicles," IEEE Electrification Magazine, vol. 1, no. 1, pp. 57-64, 2013.

[57] E. Waffenschmidt, "Wireless power for mobile devices," in 2011 IEEE 33rd international telecommunications energy conference (INTELEC), pp. 1-9, Amsterdam, Netherlands, 2011.
[58] I.-J. Yoon and H. Ling, "Investigation of near-field wireless power transfer under multiple transmitters," IEEE Antennas and Wireless Propagation Letters, vol. 10, pp. 662-665, 2011.

[59] J. J. Casanova, Zhen Ning Low, and Jenshan Lin, "A loosely coupled planar wireless power system for multiple receivers," IEEE Transactions on Industrial Electronics, vol. 56, no. 8, pp. 3060-3068, 2009.

[60] G. Dumphart, E. Slottke, and A. Wittneben, "Magnetoinductive passive relaying in arbitrarily arranged networks," in 2017 IEEE International Conference on Communications (ICC), pp. 1-6, Paris, France, 2017.

[61] X. Zhang, S. L. Ho, and W. N. Fu, "Quantitative design and analysis of relay resonators in wireless power transfer system," IEEE Transactions on Magnetics, vol. 48, no. 11, pp. 4026-4029, 2012.

[62] S. Cheon, Y.-H. Kim, S.-Y. Kang, M. L. Lee, and T. Zyung, "Wireless energy transfer system with multiple coils via coupled magnetic resonances," ETRI Journal, vol. 34, no. 4, pp. 527-535, 2012.

[63] S. Kisseleff, I. F. Akyildiz, and W. Gerstacker, "Beamforming for magnetic induction based wire-less power transfer systems with multiple receivers," in 2015 IEEE Global Communications Conference (GLOBECOM), pp. 1-7, San Diego, CA, USA, 2015.

[64] M. R. Moghadam and R. Zhang, "Multiuser wireless power transfer via magnetic resonant coupling: performance analysis, charging control, and power region characterization," IEEE transactions on signal and information processing over Networks, vol. 2, no. 1, pp. 72-83, 2015.

[65] G. Yang, M. R. V. Moghadam, and R. Zhang, "Magnetic MIMO signal processing and optimization for wireless power transfer," IEEE Transactions on Signal Processing, vol. 65, no. 11, pp. 2860-2874, 2017.

[66] H. Sun, H. Lin, F. Zhu, and F. Gao, "Magnetic resonant beamforming for secured wireless power transfer," IEEE Signal Processing Letters, vol. 24, no. 8, pp. 1173-1177, 2017.

[67] M. Kesler, "Highly resonant wireless power transfer: safe, efficient, and over distance," Witricity Corporation, 2013.

[68] D. Zhao, Z. Zhou, S. Wang, B. Liu, and W. Gaaloul, "Reinforcement learning-enabled efficient data gathering in underground wireless sensor networks," Personal and Ubiquitous Computing, pp. 1-18, 2020.

[69] S. Kisseleff, I. F. Akyildiz, and W. H. Gerstacker, "Magnetic induction-based simultaneous wireless information and power transfer for single information and multiple power receivers," IEEE Transactions on Communications, vol. 65, no. 3, pp. 1396-1410, 2017.

[70] Z. B. Popović and B. D. Popović, Introductory Electromagnetics, Addison-Wesley, 2000.

[71] M. C. Domingo, "Magnetic induction for underwater wireless communication networks," IEEE Transactions on Antennas and Propagation, vol. 60, no. 6, pp. 2929-2939, 2012.

[72] I. F. Akyildiz, P. Wang, and Z. Sun, "Realizing underwater communication through magnetic induction," IEEE Communications Magazine, vol. 53, no. 11, pp. 42-48, 2015.

[73] H. Guo, Z. Sun, and P. Wang, "Multiple frequency band channel modeling and analysis for magnetic induction communication in practical underwater environments," IEEE Transactions on Vehicular Technology, vol. 66, no. 8, pp. 6619-6632, 2017. 
[74] Y. Wang, A. Dobbin, and J. F. Bousquet, "A compact low-power underwater magneto-inductive modem," in Proceedings of the 11th ACM International Conference on Underwater Networks \& Systems, pp. 1-5, Shanghai, China, 2016.

[75] X. Tan, Z. Sun, and P. Wang, "On localization for magnetic induction-based wireless sensor networks in pipeline environments," in 2015 IEEE International Conference on Communications (ICC), pp. 2780-2785, London, UK, 2015.

[76] H. Guo and Z. Sun, "Channel and energy modeling for selfcontained wireless sensor networks in oil reservoirs," IEEE Transactions on Wireless Communications, vol. 13, no. 4, pp. 2258-2269, 2014.

[77] A. A. Alshehri, S.-C. Lin, and I. F. Akyildiz, "Optimal energy planning for wireless self-contained sensor networks in oil reservoirs," in 2017 IEEE International Conference on Communications (ICC), pp. 1-7, Paris, France, 2017.

[78] D. Abdorahimi and A. M. Sadeghioon, "Comparison of radio frequency path loss models in soil for wireless underground sensor networks," Journal of Sensor and Actuator Networks, vol. 8, no. 2, p. 35, 2019.

[79] I. F. Akyildiz, Z. Sun, and M. C. Vuran, "Signal propagation techniques for wireless underground communication networks," Physical Communication, vol. 2, no. 3, pp. 167-183, 2009.

[80] Z. Sun and I. F. Akyildiz, "On capacity of magnetic induction-based wireless underground sensor networks," in 2012 Proceedings IEEE INFOCOM, pp. 370-378, Orlando, FL, USA, 2012.

[81] E. E. Kriezis, T. D. Tsiboukis, S. M. Panas, and J. A. Tegopoulos, "Eddy currents: theory and applications," Proceedings of the IEEE, vol. 80, no. 10, pp. 1559-1589, 1992.

[82] J. R. Wait, "Mutual coupling of loops lying on the ground," Geophysics, vol. 19, no. 2, pp. 290-296, 1954.

[83] S. Kisseleff, I. F. Akyildiz, and W. H. Gerstacker, "Digital signal transmission in magnetic induction based wireless underground sensor networks," IEEE Transactions on Communications, vol. 63, no. 6, pp. 2300-2311, 2015.

[84] S. Kisseleff, B. Sackenreuter, I. F. Akyildiz, and W. Gerstacker, "On capacity of active relaying in magnetic induction based wireless underground sensor networks," in 2015 IEEE International Conference on Communications (ICC), pp. 65416546, London, UK, 2015.

[85] J. M. H. Hendrickx, B. Borchers, D. L. Corwin, S. M. Lesch, A. C. Hilgendorf, and J. Schlue, "Inversion of soil conductivity profiles from electromagnetic induction measurements: theory and experimental verification," Soil Science Society of America Journal, vol. 66, no. 3, pp. 673-685, 2002.

[86] Z. Zhang, E. Liu, Q. Xinyu, R. Wang, H. Ma, and Z. Sun, "Connectivity of magnetic induction-based ad hoc networks," IEEE Transactions on Wireless Communications, vol. 16, no. 7, pp. 4181-4191, 2017.

[87] T. E. Abrudan, O. Kypris, N. Trigoni, and A. Markham, "Impact of rocks and minerals on underground magnetoinductive communication and localization," IEEE Access, vol. 4, pp. 3999-4010, 2016.

[88] W. Steven Kisseleff, R. S. Gerstacker, Z. Sun, and I. F. Akyildiz, "Channel capacity of magnetic induction based wireless underground sensor networks under practical constraints," in 2013 IEEE Wireless Communications and Net- working Conference (WCNC), pp. 2603-2608, Shanghai, China, 2013.

[89] A. R. Silva and M. Moghaddam, "Strategic frequency adaptation for mid-range magnetic induction-based wireless underground sensor networks," in 2015 Annual IEEE Systems Conference (SysCon) Proceedings, pp. 758-765, Vancouver, BC, Canada, 2015.

[90] S. Kisseleff, I. F. Akyildiz, and W. Gerstacker, "Interference polarization in magnetic induction based wireless underground sensor networks," in 2013 IEEE 24th International Symposium on Personal, Indoor and Mobile Radio Communications (PIMRC Workshops), pp. 71-75, London, UK, 2013.

[91] E. Shamonina, V. A. Kalinin, K. H. Ringhofer, and L. Solymar, "Magneto-inductive waveguide," Electronics letters, vol. 38, no. 8, pp. 371-373, 2002.

[92] H. Guo, Z. Sun, J. Sun, and N. M. Litchinitser, " $\mathrm{M}^{2} \mathrm{I}$ : channel modeling for metamaterial-enhanced magnetic induction communications," IEEE Transactions on Antennas and Propagation, vol. 63, no. 11, pp. 5072-5087, 2015.

[93] U. Mengali, Synchronization Techniques for Digital Receivers, Springer Science \& Business Media, 2013.

[94] S. C. Ergen and P. Varaiya, "TDMA scheduling algorithms for wireless sensor networks," Wireless Networks, vol. 16, no. 4, pp. 985-997, 2010.

[95] S. Kisseleff, I. F. Akyildiz, and W. Gerstacker, “Transmitterside channel estimation in magnetic induction based communication systems," in 2014 IEEE International Black Sea Conference on Communications and Networking (Black SeaCom), pp. 16-21, Odessa, Ukraine, 2014.

[96] S. Kisseleff, I. F. Akyildiz, and W. Gerstacker, "Disaster detection in magnetic induction based wireless sensor networks with limited feedback," in 2014 IFIP Wireless Days (WD), pp. 1-7, Rio de Janeiro, Brazil, 2014.

[97] Z. Sun, I. F. Akyildiz, S. Kisseleff, and W. Gerstacker, "Increasing the capacity of magnetic induction communications in RF-challenged environments," IEEE Transactions on Communications, vol. 61, no. 9, pp. 3943-3952, 2013.

[98] Z. Sun and I. F. Akyildiz, "Optimal deployment for magnetic induction-based wireless networks in challenged environments," IEEE Transactions on Wireless Communications, vol. 12, no. 3, pp. 996-1005, 2013.

[99] P. Gupta and P. R. Kumar, "The capacity of wireless networks," IEEE Transactions on Information Theory, vol. 46, no. 2, pp. 388-404, 2000.

[100] S. Kisseleff, I. F. Akyildiz, and W. H. Gerstacker, "Throughput of the magnetic induction based wireless underground sensor networks: key optimization techniques," IEEE Transactions on Communications, vol. 62, no. 12, pp. 4426-4439, 2014.

[101] S. Kisseleff, X. Chen, I. F. Akyildiz, and W. Gerstacker, "Localization of a silent target node in magnetic induction based wireless underground sensor networks," in 2017 IEEE International Conference on Communications (ICC), pp. 17, Paris, France, 2017.

[102] N. Ahmed, Y. R. Zheng, and D. Pommerenke, "Multi-coil mi based mac protocol for wireless sensor networks," in OCEANS 2016 MTS/IEEE Monterey, pp. 1-4, Monterey, CA, USA, 2016.

[103] A. Gungi, K. A. Vikas Vippalapalli, U. Menon, and B. Hariharan, "Inductively powered underground wireless 
communication system," in Microelectronics, Electromagnetics and Telecommunications, pp. 205-215, Springer, 2016.

[104] S. Olariu and I. Stojmenovic, "Design guidelines for maximizing lifetime and avoiding energy holes in sensor networks with uniform distribution and uniform reporting," in Proceedings IEEE INFOCOM 2006. 25TH IEEE International Conference on Computer Communications, pp. 1-12, Rennes, France, 2006.

[105] N. T. Tam, H. T. T. Binh, D. A. Dung et al., "A hybrid clustering and evolutionary approach for wireless underground sensor network lifetime maximization," Information Sciences, vol. 504, pp. 372-393, 2019.

[106] N. Mittal, U. Singh, R. Salgotra, and M. Bansal, “An energyefficient stable clustering approach using fuzzy-enhanced flower pollination algorithm for WSNs," Neural Computing and Applications, vol. 32, no. 11, pp. 7399-7419, 2020.

[107] L. Xie, Y. Yi Shi, T. Hou, and A. Lou, "Wireless power transfer and applications to sensor networks," IEEE Wireless Communications, vol. 20, no. 4, pp. 140-145, 2013.

[108] J. Pan, B. Xue, and Y. Inoue, “A self-powered sensor module using vibration-based energy generation for ubiquitous systems," in 2005 6th International Conference on ASIC, vol. 1, pp. 403-406, Shanghai, 2005.

[109] A. A. Alshehri, FracBot: design of wireless underground sensor networks for mapping hydraulic fractures and determining reservoir parameters in unconventional systems, $\mathrm{PhD}$ thesis, Georgia Institute of Technology, 2018.

[110] P. Singh and N. Mittal, "Efficient localisation approach for WSNs using hybrid DA-FA algorithm," IET Communications, vol. 14, no. 12, pp. 1975-1991, 2020.

[111] K. Mori, "Application of weight functions to the magnetic localization of an object," IEEE transac tions on magnetics, vol. 25, no. 3, pp. 2726-2731, 1989.

[112] E. A. Prigge and J. P. How, "Signal architecture for a distributed magnetic local positioning system," IEEE Sensors Journal, vol. 4, no. 6, pp. 864-873, 2004.

[113] A. Markham, N. Trigoni, S. A. Ellwood, and D. W. Macdonald, "Revealing the hidden lives of underground animals using magneto-inductive tracking," in Proceedings of the 8th ACM conference on embedded networked sensor systems, pp. 281-294, Zürich, Switzerland, 2010.

[114] M. A. Akkaş, "Using wireless underground sensor networks for mine and miner safety," Wireless Networks, vol. 24, no. 1, pp. 17-26, 2018.

[115] S. R. Rusu, M. J. Hayes, and J. A. Marshall, "Localization in large-scale underground environments with RFID," in 2011 24th Canadian Conference on Electrical and Computer Engineering (CCECE), pp. 001140-001143, Niagara Falls, ON, Canada, 2011.

[116] J. Ma, X. Zhang, Q. Huang, L. Cheng, and L. Mingyu, "Experimental study on the impact of soil conductivity on underground magneto-inductive channel," IEEE Antennas and Wireless Propagation Letters, vol. 14, pp. 1782-1785, 2015.

[117] A. Markham and N. Trigoni, "Magneto-inductive networked rescue system (miners) taking sensor networks underground," in Proceedings of the 11th international conference on Information Processing in Sensor Networks, pp. 317-328, Beijing, China, 2012.

[118] J. Wan, S. Tang, Z. Shu et al., "Software-defined industrial Internet of things in the context of Industry 4.0," IEEE Sensors Journal, vol. 16, pp. 7373-7380, 2016.
[119] J. Heidemann, M. Stojanovic, and M. Zorzi, "Underwater sensor networks: applications, advances and challenges," Philosophical Transactions of the Royal Society A: Mathematical, Physical and Engineering Sciences, vol. 370, pp. 158-175, 2012.

[120] R. W. Coutinho, A. Boukerche, L. F. Vieira, and A. A. Loureiro, "On the design of green protocols for underwater sensor networks," IEEE Communications Magazine, vol. 54, no. 10, pp. 67-73, 2016.

[121] S. Jain, A. Kumar, S. Mandal et al., "B4: experience with a globally-deployed software defined WAN," ACM SIGCOMM Computer Communication Review, vol. 43, no. 4, pp. 3-14, 2013. 